International Journal of Pure and Applied Mathematics

Volume 96 No. 3 2014, 391-405

ISSN: 1311-8080 (printed version); ISSN: 1314-3395 (on-line version)

url: http://www.ijpam.eu

doi: http://dx.doi.org/10.12732/ijpam.v96i3.9

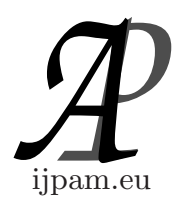

\title{
ON CERTAIN ESTIMATES FOR PARABOLIC MARCINKIEWICZ INTEGRAL AND EXTRAPOLATION
}

\author{
Mohammed Ali ${ }^{1}$, Eman Abo-Shgair ${ }^{2}$ \\ ${ }^{1,2}$ Department of Mathematics and Statistics \\ Jordan University of Science and Technology \\ Irbid, JORDAN
}

\begin{abstract}
In this article, we establish $L^{p}$ boundedness of the parametric Marcinkiewicz integral operators with rough kernels. These estimates and extrapolation arguments improve and extend some known results on parabolic Marcinkiewicz integrals.
\end{abstract}

AMS Subject Classification: 40B15, 40B20, 40B25

Key Words: $L^{p}$ boundedness, prabolic Marcinkiewicz integrals, rough kernels, extrapolation

\section{Introduction}

Throughout this article, let $\mathbf{R}^{n}, n \geq 2$, be the $n$-dimensional Euclidean space, and let $\mathbf{S}^{n-1}$ be the unit sphere in $\mathbf{R}^{n}$ which is equipped with the normalized Lebesgue surface measure $d \sigma=d \sigma(\cdot)$. Also, let $p^{\prime}$ denote to the exponent conjugate to $p$; that is $1 / p+1 / p^{\prime}=1$.

Let $\alpha_{i} \geq 1,(i=1,2, \cdots, n)$, be fixed real numbers. For fixed $x \in \mathbf{R}^{n}$ and $\rho>0$, let $F(x, \rho)=\sum_{i=1}^{n} \frac{x_{i}^{2}}{\rho^{2 \alpha_{i}}}$. Then it is easy to see that for any fixed $x \in \mathbf{R}^{n} \backslash\{0\}, F(x, \rho)$ is strictly decreasing function in $\rho>0$. The unique solution of the equation $F(x, \rho)=1$ is denoted by $\rho(x)$.It was proved in [13]

Received: August 21, 2014

(C) 2014 Academic Publications, Ltd.

$\S_{\text {Correspondence author }}$ url: www.acadpubl.eu 
that $\rho(x)$ is a metric on $\mathbf{R}^{n}$, and $\left(\mathbf{R}^{n}, \rho\right)$ is the mixed homogeneity space.

$$
\text { For } \lambda>0 \text {, let } A_{\lambda}=\left[\begin{array}{ccc}
\lambda^{\alpha_{1}} & & 0 \\
& \ddots & \\
0 & & \lambda^{\alpha_{n}}
\end{array}\right] \text {. For } \tau=a+i b(a, b \in \mathbf{R} \text { with } a>0) \text {, }
$$

let $K_{\Omega, h}(u)=\Omega(u) h(\rho(u)) \rho(u)^{\tau-\alpha}$, where $h:[0, \infty) \rightarrow \mathbf{C}$ is a measurable function and $\Omega$ is a real valued and measurable function on $\mathbf{R}^{n}$ with $\Omega \in$ $L^{1}\left(\mathbf{S}^{n-1}\right)$ that satisfying the conditions

$$
\begin{array}{r}
\Omega\left(A_{\lambda} x\right)=\Omega(x), \text { and } \\
\int_{\mathbf{S}^{n-1}} \Omega\left(x^{\prime}\right) J\left(x^{\prime}\right) d \sigma\left(x^{\prime}\right)=0,
\end{array}
$$

where $J\left(x^{\prime}\right)$ is a function will be defined later. Define the parabolic Marcinkiewicz integral operator $\mathcal{M}_{\Omega, h}^{\tau}$ for $f \in \mathcal{S}\left(\mathbf{R}^{n}\right)$ by

$$
\mathcal{M}_{\Omega, h}^{\tau} f(x)=\left(\int_{0}^{\infty}\left|\frac{1}{t^{\tau}} \int_{\rho(u) \leq t} K_{\Omega, h}(u) f(x-u) d u\right|^{2} \frac{d t}{t}\right)^{1 / 2} .
$$

When $\tau=1$ and $h=1$, we denote $\mathcal{M}_{\Omega, h}^{\tau}$ by $\mu_{\Omega}$. The parabolic LittlewoodPaley operator $\mu_{\Omega}$ was introduced by Xue, Ding and Yabuta in [20] in which they proved that $\mu_{\Omega}$ is bounded for $p \in(1, \infty)$ provided that $\Omega \in L^{q}\left(\mathbf{S}^{n-1}\right)$ for $q>1$. Subsequently, the study of the $L^{p}$ boundedness of $\mu_{\Omega}$ under various conditions on the function $\Omega$ has been studied by many authors. For example, Cheng and Ding improved the above result in [8]; they obtained the $L^{p}$ boundedness of $\mu_{\Omega}$ when $\Omega$ belongs to the Hardy space $H^{1}\left(\mathbf{S}^{n-1}\right)$ for $1<p<\infty$. However, the authors of [6] established that $\mu_{\Omega}$ is bounded under the condition $\Omega \in L(\log L)^{1 / 2}\left(\mathbf{S}^{n-1}\right)$ for $1<p<\infty$. In addition, the authors of [10] found that $\mu_{\Omega}$ is bounded when $\Omega$ belongs to the block space $B_{q}^{(0,-1 / 2)}\left(\mathbf{S}^{n-1}\right)$ for $1<p<\infty$ and $q>1$. Recently, Wang, Chen and Yu verified in [19] that if we replace $n$ by $n+1$, and $y$ by $(y, \phi(\rho(y)))$, where $\phi$ is a polynomial of degree $m$, then $\mu_{\Omega}$ is bounded on $L^{p}\left(\mathbf{R}^{n+1}\right)$ for $p \in\left(\frac{2+2 \nu}{1+2 \nu}, 2+2 \nu\right)$ provided that $\Omega \in F\left(\nu, \mathbf{S}^{n-1}\right)$ for some $\nu>0$, where $F\left(\nu, \mathbf{S}^{n-1}\right)$ denotes the set of all $\Omega$ which are integrable over $\mathbf{S}^{n-1}$ and satisfying

$$
\sup _{\xi \in \mathbf{S}^{n-1}} \int_{\mathbf{S}^{n-1}}|\Omega(u)|\left(\ln \frac{1}{|u \cdot \xi|}\right)^{1+\nu} d u<\infty .
$$


We point out that the class of the operators $\mu_{\Omega}$ is related to the class of the parabolic singular integral operators

$$
T_{\Omega} f(x)=p \cdot v \cdot \int_{\mathbf{R}^{n}} \frac{\Omega(u)}{\rho(u)^{\alpha}} f(x-u) d u .
$$

The class of the operators $T_{\Omega}$ belongs to the class of singular Radon transforms, which has considered to study by many mathematicians (we refer the readers, in particular, to [13], [15] and [16]).

If $\alpha_{1}=\cdots=\alpha_{n}=1$, then $\rho(x)=|x|, \alpha=n$ and $\left(\mathbf{R}^{n}, \rho\right)=\left(\mathbf{R}^{n},|\cdot|\right)$. In this case, $\mu_{\Omega}$ is just the classical Marcinkiewicz integral, which was introduced by Stein in [18]. For more information about the importance and the recent advances on the study of such operators, the readers are refereed (for instance to $[2],[3],[5],[9],[11],[12],[14]$, and the references therein).

Our main interest in this paper is to study the $L^{p}$ boundedness of the parabolic Marcinkiewicz integral under weak conditions on $\Omega$ and $h$, and then apply an extrapolation method to establish new improved results. In this work, we let $\Delta_{\gamma}\left(\mathbf{R}^{+}\right)$( for $\gamma>1$ ) denote the collection of all measurable functions $h:[0, \infty) \rightarrow \mathbf{C}$ satisfying

$$
\|h\|_{\Delta_{\gamma}\left(\mathbf{R}^{+}\right)}=\sup _{R \in \mathbf{Z}}\left(\frac{1}{R} \int_{0}^{R}|h(\rho)|^{\gamma} d \rho\right)^{1 / \gamma}<\infty .
$$

In this article, we extend and improve some known results in the parabolic Marcinkiewicz operators (see [1], [6], [10], and [20]). Our main result is formulated as follows:

Theorem 1. Let $\Omega \in L^{q}\left(\mathbf{S}^{n-1}\right)$ for some $1<q \leq 2$ and $h \in \triangle_{\gamma}\left(\boldsymbol{R}^{+}\right)$ for some $\gamma>1$. Then for any $f \in L^{p}\left(\mathbf{R}^{n}\right)$ with $p$ satisfying $|1 / p-1 / 2|<$ $\min \left\{1 / 2,1 / \gamma^{\prime}\right\}$, a constant $C_{p}$ (independent of $\Omega, h, \gamma$, and $q$ ) exists such that

$$
\left\|\mathcal{M}_{\Omega, h}^{\tau} f\right\|_{L^{p}\left(\mathbf{R}^{n}\right)} \leq C_{p} A(\gamma)(q-1)^{-1 / 2}\|h\|_{\Delta_{\gamma}\left(\mathbf{R}^{+}\right)}\|\Omega\|_{L^{q}\left(\mathbf{S}^{n-1}\right)}\|f\|_{L^{p}\left(\mathbf{R}^{n}\right)},
$$

where $A(\gamma)= \begin{cases}\gamma^{1 / 2} & \text { if } \gamma>2, \\ (\gamma-1)^{-1 / 2} & \text { if } 1<\gamma \leq 2 .\end{cases}$

The fruit of our result is earned by using its conclusion and the extrapolation method (see [4]). In particular, Theorem 1 and extrapolation lead to the following theorem.

Theorem 2. Suppose that $\Omega$ satisfies the conditions (1)-(2) and $h \in$ $\triangle_{\gamma}\left(\boldsymbol{R}^{+}\right)$for some $\gamma>1$. 
(i) If $\Omega \in B_{q}^{(0,-1 / 2)}\left(\mathbf{S}^{n-1}\right)$ for some $q>1$, then for $|1 / p-1 / 2|<\min \left\{1 / 2,1 / \gamma^{\prime}\right\}$, $\left\|\mathcal{M}_{\Omega, h}^{\tau} f\right\|_{L^{p}\left(\mathbf{R}^{n}\right)} \leq C_{p} A(\gamma)\|h\|_{\triangle_{\gamma}\left(\boldsymbol{R}^{+}\right)}\|f\|_{L^{p}\left(\boldsymbol{R}^{n}\right)}\left(1+\|\Omega\|_{B_{q}^{(0,-1 / 2)}\left(\mathbf{S}^{n-1}\right)}\right) ;$

(ii) If $\Omega \in L(\log L)^{1 / 2}\left(\mathbf{S}^{n-1}\right)$, then

$$
\left\|\mathcal{M}_{\Omega, h}^{\tau} f\right\|_{L^{p}\left(\mathbf{R}^{n}\right)} \leq C_{p} A(\gamma)\|h\|_{\triangle_{\gamma}\left(\boldsymbol{R}^{+}\right)}\|f\|_{L^{p}\left(\boldsymbol{R}^{n}\right)}\left(1+\|\Omega\|_{L(\log L)^{1 / 2}\left(\mathbf{S}^{n-1}\right)}\right)
$$

for $|1 / p-1 / 2|<\min \left\{1 / 2,1 / \gamma^{\prime}\right\}$.

Throughout this paper, the letter $C$ denotes a bounded positive constant that may vary at each occurrence but independent of the essential variables.

\section{Some Lemmas}

In this section, we give some auxiliary lemmas used in the sequel. Let us first recall the polar coordinates transform in the mixed homogeneity space $\left(\mathbf{R}^{n}, \rho\right)$. For $x=\left(x_{1}, x_{2}, \cdots, x_{n}\right) \in \mathbf{R}^{n}$, set

$$
\begin{aligned}
& x_{1}=\rho^{\alpha_{1}} \cos \vartheta_{1} \cdots \cos \vartheta_{n-2} \cos \vartheta_{n-1}, \\
& x_{2}=\rho^{\alpha_{2}} \cos \vartheta_{1} \cdots \cos \vartheta_{n-2} \sin \vartheta_{n-1}, \\
& \vdots \\
& x_{n-1}=\rho^{\alpha_{n-1}} \cos \vartheta_{1} \sin \vartheta_{2}, \\
& x_{n}=\rho^{\alpha_{n}} \sin \vartheta_{1} .
\end{aligned}
$$

Thus, $d x=\rho^{\alpha-1} J\left(\vartheta_{1}, \cdots, \vartheta_{n-1}\right) d \rho d \sigma\left(x^{\prime}\right)$, where $\rho^{\alpha-1} J\left(\vartheta_{1}, \cdots, \vartheta_{n-1}\right)$ is the Jacobian of the above transforms. It was shown in [13] that $J\left(\vartheta_{1}, \cdots, \vartheta_{n-1}\right)$ is a $C^{\infty}\left((0,2 \pi)^{n-2} \times(0, \pi)\right)$ function in the variable $x^{\prime} \in \mathbf{S}^{n-1}$, and that a real constant $M \geq 1$ exists so that $1 \leq J\left(\vartheta_{1}, \cdots, \vartheta_{n-1}\right) \leq M$. For simplicity, we let $J\left(x^{\prime}\right)$ denote $J\left(\vartheta_{1}, \cdots, \vartheta_{n-1}\right)$.

In order to prove Theorem 1, we need the following lemmas.

Lemma 3. [17]. Suppose that $\lambda_{i}^{\prime} s$ and $\alpha_{i}^{\prime} s$ are fixed real numbers, and $\Gamma(t)=\left(\lambda_{1} t^{\alpha_{1}}, \cdots, \lambda_{n} t^{\alpha_{n}}\right)$ is a function from $\boldsymbol{R}^{+}$to $\boldsymbol{R}^{n}$. For suitable $f$, let $\mathcal{M}_{\Gamma}$ be the maximal operator defined on $\boldsymbol{R}^{n}$ by

$$
\mathcal{M}_{\Gamma}(f)(x)=\sup _{h>0} \frac{1}{h}\left|\int_{0}^{h} f(x-\Gamma(t)) d t\right|
$$

for $x \in \boldsymbol{R}^{n}$. Then for $1<p \leq \infty$, there exists a constant $C_{p}>0$ such that

$$
\left\|\mathcal{M}_{\Gamma}(f)\right\|_{L^{p}\left(\boldsymbol{R}^{n}\right)} \leq C_{p}\|f\|_{L^{p}\left(\boldsymbol{R}^{n}\right)} .
$$

The constant $C_{p}$ is independent of $\lambda_{i}^{\prime} s$ and $f$. 
Lemma 4. [6]. Let $\beta \in[0,1]$. Then for any $u, \xi \in \boldsymbol{R}^{n}$,

$$
\left|\int_{1}^{2} e^{-i A_{\lambda} u \cdot \xi} \frac{d \lambda}{\lambda}\right| \leq C|u \cdot \xi|^{-\frac{\beta}{m}}
$$

where $A_{\lambda}$ is defined as above and $m$ denotes the distinct numbers of $\left\{\alpha_{i}\right\}$.

We shall recall the following lemma due to Chen and Ding.

Lemma 5. [7]. Let $\phi \in \mathcal{S}\left(\boldsymbol{R}^{n}\right), \widehat{\Phi}(\xi)=\phi(\rho(\xi))$ and $\Phi_{t}(\xi)=t^{-\alpha} \phi\left(A_{t^{-1}} \xi\right)$ for $t>0$. For $j \in \boldsymbol{Z}$, define the multiplier $\widehat{S_{j} f}(\xi)=\phi\left(2^{j} \rho(\xi)\right) \hat{f}(\xi)$. Then for $1<p<\infty$, there exists a constant $C$ such that

$$
\left\|\left(\sum_{j}\left|S_{j} f\right|^{2}\right)^{1 / 2}\right\|_{L^{p}\left(\boldsymbol{R}^{n}\right)} \leq C\|f\|_{L^{p}\left(\boldsymbol{R}^{n}\right)} .
$$

Let $\theta \geq 2$. For a measurable function $h: \mathbf{R}^{+} \rightarrow \mathbf{C}$ and $\Omega: \mathbf{S}^{n-1} \rightarrow \mathbf{R}$, we define the family of measures $\left\{\sigma_{\Omega, h, t}: t \in \mathbf{R}^{+}\right\}$and its corresponding maximal operators $\sigma_{\Omega, h, t}^{*}$ and $M_{h, \theta}$ on $\mathbf{R}^{n}$ by

$$
\begin{gathered}
\int_{\mathbf{R}^{n}} f d \sigma_{\Omega, h, t}=\frac{1}{t^{\tau}} \int_{1 / 2 t \leq \rho(u) \leq t} f(u) \frac{h(\rho(u)) \Omega(u)}{\rho(u)^{\alpha-\tau}} d u, \\
\sigma_{\Omega, h}^{*} f(x)=\sup _{t \in \mathbf{R}^{+}}|| \sigma_{\Omega, h, t}|* f(x)|,
\end{gathered}
$$

and

$$
M_{h, \theta} f(x)=\sup _{k \in \mathbf{Z}} \int_{\theta^{k}}^{\theta^{k+1}}|| \sigma_{\Omega, h, t}|* f(x)| \frac{d t}{t},
$$

where $\left|\sigma_{\Omega, h, t}\right|$ is defined in the same way as $\sigma_{\Omega, h, t}$, but with replacing $h$ by $|h|$ and $\Omega$ by $|\Omega|$. We write $\left\|\nu_{t, s}\right\|$ for the total variation of $\nu_{t, s}$.

Lemma 6. Let $\theta \geq 2, h \in \Delta_{\gamma}\left(\mathbf{R}^{+}\right)$for some $\gamma>1, \Omega \in L^{q}\left(\mathbf{S}^{n-1}\right)$ for some $q>1$. Then there exist constants $C$ and $\beta$ with $0<\beta<\min \left\{\frac{1}{2}, \frac{m}{2 q^{\prime}}, \frac{m}{\alpha}\right\}$ such that

$$
\left\|\sigma_{\Omega, h, t}\right\| \leq C(\ln \theta)\|h\|_{\Delta_{\gamma}\left(\mathbf{R}^{+}\right)}^{2}\|\Omega\|_{L^{q}\left(\mathbf{S}^{n-1}\right)}^{2} ;
$$




$$
\int_{\theta^{k}}^{\theta^{k+1}}\left|\hat{\sigma}_{\Omega, h, t}(\xi)\right|^{2} \frac{d t}{t} \leq C(\ln \theta)\left|\xi A_{\theta^{k}}\right|^{ \pm \frac{2 \beta}{m q^{\prime} \gamma^{\prime}}}\|h\|_{\Delta_{\gamma}\left(\mathbf{R}^{+}\right)}^{2}\|\Omega\|_{L^{q}\left(\mathbf{S}^{n-1}\right)}^{2}
$$

hold for all $k \in \mathbf{Z}$, where $\left|\xi A_{\theta^{k}}\right|^{ \pm \frac{2 \beta}{m q^{\prime} \gamma^{\prime}}}=\inf \left\{\left|\xi A_{\theta^{k}}\right|^{+\frac{2 \beta}{m q^{\prime} \gamma^{\prime}}},\left|\xi A_{\theta^{k}}\right|^{-\frac{2 \beta}{\left.m q^{\prime} \gamma^{\prime}\right)}}\right\}$. The constant $C$ is independent of $k$ and $\xi$.

Proof. We prove this lemma only for the case $1<q \leq 2$, since $L^{q}\left(\mathbf{S}^{n-1}\right) \subseteq$ $L^{2}\left(\mathbf{S}^{n-1}\right)$ for $q \geq 2$. By Hölder's inequality, we obtain that

$$
\begin{aligned}
\left|\hat{\sigma}_{\Omega, h, t}(\xi)\right| & \leq \int_{\frac{1}{2} t}^{t}|h(\rho)|\left|\int_{\mathbf{S}^{n-1}} e^{-i A_{\rho} x \cdot \xi} \Omega(x) J(x) d \sigma(x)\right| \frac{d \rho}{\rho} \\
& \leq\|h\|_{\Delta_{\gamma}\left(\mathbf{R}^{+}\right)}\left(\int_{\frac{1}{2} t}^{t}\left|\int_{\mathbf{S}^{n-1}} e^{-i A_{\rho} \xi \cdot x} \Omega(x) J(x) d \sigma(x)\right|^{\gamma^{\prime}} \frac{d \rho}{\rho}\right)^{1 / \gamma^{\prime}} .
\end{aligned}
$$

On one hand, if $1<\gamma \leq 2$, then by a change of variable we get that

$$
\begin{aligned}
\left|\hat{\sigma}_{\Omega, h, t}(\xi)\right| & \leq\|h\|_{\Delta_{\gamma}\left(\mathrm{R}^{+}\right)}\|\Omega\|_{L^{1}\left(\mathbf{S}^{n-1}\right)}^{\left(1-2 / \gamma^{\prime}\right)}\left(\int_{\frac{1}{2} t}^{t}\left|\int_{\mathbf{S}^{n-1}} e^{-i A_{\rho} \xi \cdot x} \Omega(x) J(x) d \sigma(x)\right|^{2} \frac{d \rho}{\rho}\right)^{1 / \gamma^{\prime}} \\
& \leq C\|h\|_{\Delta_{\gamma}\left(\mathrm{R}^{+}\right)}\|\Omega\|_{L^{1}\left(\mathbf{S}^{n-1}\right)}^{\left(1-2 / \gamma^{\prime}\right)}\left(\int_{\mathbf{S}^{n-1} \times \mathbf{S}^{n-1}} \Omega(x) \overline{\Omega(y)} I(\xi, x, y) d \sigma(x) d \sigma(y)\right)^{1 / \gamma^{\prime}},
\end{aligned}
$$

where $I(\xi, x, y)=\int_{1}^{2} e^{-i A_{\frac{t}{2}} \xi \cdot(x-y)} \frac{d \rho}{\rho}$. Let $\eta=\frac{A_{\frac{t}{2}} \xi}{\left|A_{\frac{t}{2}} \xi\right|}$. Since $\beta<m / \alpha$, then by lemma 4 we get that

$$
\begin{aligned}
I(\xi, x, y) & \leq C\left|A_{\frac{t}{2}} \xi \cdot(x-y)\right|^{-\beta / m}=C 2^{\alpha \beta / m} \mid\left(\left|\eta \cdot(x-y) \| A_{t} \xi\right|\right)^{-\beta / m} \\
& \leq C\left|A_{t} \xi\right|^{-\beta / m}(|\eta \cdot(x-y)|)^{-\beta / m}
\end{aligned}
$$

which leads to

$$
\left|\hat{\sigma}_{\Omega, h, t}(\xi)\right| \leq C\|h\|_{\Delta_{\gamma}\left(\mathbf{R}^{+}\right)}\|\Omega\|_{L^{1}\left(\mathbf{S}^{n-1}\right)}^{\left(1-2 / \gamma^{\prime}\right)}
$$




$$
\begin{aligned}
& \times\left(\int_{\mathbf{S}^{n-1} \times \mathbf{S}^{n-1}} \Omega(x) \overline{\Omega(y)}\left(\left|\eta \cdot(x-y) \| A_{t} \xi\right|\right)^{-\beta / m} d \sigma(x) d \sigma(y)\right)^{1 / \gamma^{\prime}} \\
& \leq C\|h\|_{\Delta_{\gamma}\left(\mathbf{R}^{+}\right)}\|\Omega\|_{L^{1}\left(\mathbf{S}^{n-1}\right)}^{\left(1-2 / \gamma^{\prime}\right)}\|\Omega\|_{L^{q}\left(\mathbf{S}^{n-1}\right)}^{2 / \gamma^{\prime}}\left|A_{t} \xi\right|^{-\beta /\left(q^{\prime} m \gamma^{\prime}\right)} \\
& \times\left(\int_{\mathbf{S}^{n-1} \times \mathbf{S}^{n-1}}(|\eta \cdot(x-y)|)^{-\beta q^{\prime} / m} d \sigma(x) d \sigma(y)\right)^{1 /\left(q^{\prime} \gamma^{\prime}\right)} \text {. }
\end{aligned}
$$

As $0<\beta<\frac{m}{2 q^{\prime}}$, we get that the last integral is finite, and hence

$$
\begin{aligned}
\left|\hat{\sigma}_{\Omega, h, t}(\xi)\right| & \leq C\|h\|_{\Delta_{\gamma}\left(\mathbf{R}^{+}\right)}\|\Omega\|_{L^{1}\left(\mathbf{S}^{n-1}\right)}^{\left(1-2 / \gamma^{\prime}\right)}\|\Omega\|_{L^{q}\left(\mathbf{S}^{n-1}\right)}^{2 / \gamma^{\prime}}\left|A_{t} \xi\right|^{-\beta /\left(q^{\prime} m \gamma^{\prime}\right)} \\
& \leq C\|h\|_{\Delta_{\gamma}\left(\mathbf{R}^{+}\right)}\|\Omega\|_{L^{q}\left(\mathbf{S}^{n-1}\right)}\left|A_{t} \xi\right|^{-\beta /\left(q^{\prime} m \gamma^{\prime}\right)}
\end{aligned}
$$

Thus, we derive

$$
\int_{\theta^{k}}^{\theta^{k+1}}\left|\hat{\sigma}_{\Omega, h, t}(\xi)\right|^{2} \frac{d t}{t} \leq C(\ln \theta)\left|A_{\theta^{k}} \xi\right|^{-\frac{2 \beta}{q^{\prime} m \gamma^{\prime}}}\|h\|_{\Delta_{\gamma}\left(\mathbf{R}^{+}\right)}^{2}\|\Omega\|_{L^{q}\left(\mathbf{S}^{n-1}\right)}^{2} .
$$

On the other hand, if $\gamma>2$, then by using Hölder's inequality, we get that

$$
\begin{aligned}
& \left|\hat{\sigma}_{\Omega, h, t}(\xi)\right| \leq C\|h\|_{\Delta_{\gamma}\left(\mathbf{R}^{+}\right)}\left(\int_{\frac{1}{2} t}^{t}\left|\int_{\mathbf{S}^{n-1}} e^{-i A_{\rho} x \cdot \xi} \Omega(x) d \sigma(x)\right|^{2} \frac{d \rho}{\rho}\right)^{1 / 2} \\
\leq & \|h\|_{\Delta_{\gamma}\left(\mathbf{R}^{+}\right)}\left(\int_{\mathbf{S}^{n-1} \times \mathbf{S}^{n-1}} \Omega(x) \overline{\Omega(y)} \int_{\frac{1}{2}}^{1} e^{-i A_{t \rho}(x-y) \cdot \xi} \frac{d \rho}{\rho} d \sigma(x) d \sigma(y)\right)^{1 / 2} .
\end{aligned}
$$

Following the above procedure give

$$
\left|\hat{\sigma}_{\Omega, h, t}(\xi)\right| \leq C\left|A_{t} \xi\right|^{-\beta /\left(q^{\prime} m \gamma^{\prime}\right)}\|h\|_{\Delta_{\gamma}\left(\mathbf{R}^{+}\right)}\|\Omega\|_{L^{q}\left(\mathbf{S}^{n-1}\right)},
$$

and therefore,

$$
\int_{\theta^{k}}^{\theta^{k+1}}\left|\hat{\sigma}_{\Omega, h, t}(\xi)\right|^{2} \frac{d t}{t} \leq C(\ln \theta)\left|A_{\theta^{k}} \xi\right|^{-2 \beta /\left(q^{\prime} m \gamma^{\prime}\right)}\|h\|_{\Delta_{\gamma}\left(\mathbf{R}^{+}\right)}^{2}\|\Omega\|_{L^{q}\left(\mathbf{S}^{n-1}\right)}^{2} .
$$


To prove the other estimate in Lemma 6, we use the cancelation property of $\Omega$.

$$
\begin{aligned}
& \int_{\theta^{k}}^{\theta^{k+1}}\left|\hat{\sigma}_{\Omega, h, t}(\xi)\right|^{2} \frac{d t}{t} \leq\|\Omega\|_{L^{1}\left(\mathbf{S}^{n-1}\right)} \int_{\mathbf{S}^{n-1}}|\Omega(x)||J(x)|^{2} \\
& \quad \times \int_{\theta^{k}}^{\theta^{k+1} \mid}\left|\int_{\frac{1}{2}}^{1}\right| e^{-i A_{t \rho} \xi \cdot x}-\left.1|| h(t \rho) \frac{d \rho}{\rho}\right|^{2} \frac{d t}{t} d \sigma(x) \\
& \leq C\|\Omega\|_{L^{1}\left(\mathbf{S}^{n-1}\right)} \int_{\mathbf{S}^{n-1}}|\Omega(x)| \int_{1}^{\theta}\left|\int_{\frac{1}{2}}^{1}\right| A_{\theta^{k} t \rho} \xi \cdot x \|\left. h\left(\theta^{k} t \rho\right) \frac{d \rho}{\rho}\right|^{2} \frac{d t}{t} d \sigma(x) \\
& \leq C\|\Omega\|_{L^{1}\left(\mathbf{S}^{n-1}\right)} \int_{\mathbf{S}^{n-1}}|\Omega(x)| \int_{1}^{\theta}\left|\int_{\frac{1}{2}}^{1}\right| A_{\theta^{k} \rho} \xi \|\left. h\left(\theta^{k} t \rho\right) \frac{d \rho}{\rho}\right|^{2} \frac{d t}{t} d \sigma(x) .
\end{aligned}
$$

Since $\gamma>1$ and $\frac{1}{2}<\rho<1$, we obtain that

$$
\int_{\theta^{k}}^{\theta^{k+1}}\left|\hat{\sigma}_{\Omega, h, t}(\xi)\right|^{2} \frac{d t}{t} \leq C(\ln \theta)\left|A_{\theta^{k}} \xi\right|^{2}\|h\|_{\Delta_{\gamma}\left(\mathbf{R}^{+}\right)}^{2}\|\Omega\|_{L^{q}\left(\mathbf{S}^{n-1}\right)}^{2},
$$

which when combine with the trivial estimate

$$
\int_{\theta^{k}}^{\theta^{k+1}}\left|\hat{\sigma}_{\Omega, h, t}(\xi)\right|^{2} \frac{d t}{t} \leq C(\ln \theta)\|h\|_{\Delta_{\gamma}\left(\mathbf{R}^{+}\right)}^{2}\|\Omega\|_{L^{q}\left(\mathbf{S}^{n-1}\right)}^{2}
$$

provides

$$
\int_{\theta^{k}}^{\theta^{k+1}}\left|\hat{\sigma}_{\Omega, h, t}(\xi)\right|^{2} \frac{d t}{t} \leq C(\ln \theta)\left|A_{\theta^{k}} \xi\right|^{\frac{2 \beta}{m q^{\prime} \gamma^{\prime}}}\|h\|_{\Delta_{\gamma}\left(\mathbf{R}^{+}\right)}^{2}\|\Omega\|_{L^{q}\left(\mathbf{S}^{n-1}\right)}^{2} .
$$

The proof is complete.

Lemma 7. Let $\Omega \in L^{q}\left(\mathbf{S}^{n-1}\right)$ for some $1<q \leq 2$ and $h \in \Delta_{\gamma}\left(\mathbf{R}^{+}\right)$for some $\gamma>1$. Then for any $f \in L^{p}\left(\mathbf{R}^{n}\right)$ with $\gamma^{\prime}<p \leq \infty$, there exists a constant $C_{p}$ (independent of $\Omega, h$ and $f$ ) such that

$$
\left\|\sigma_{\Omega, h}^{*} f(x)\right\|_{L^{p}\left(\mathbf{R}^{n}\right)} \leq C_{p}\|h\|_{\Delta_{\gamma}\left(\mathbf{R}^{+}\right)}\|\Omega\|_{L^{q}\left(\mathbf{S}^{n-1}\right)}\|f\|_{L^{p}\left(\mathbf{R}^{n}\right)} .
$$


Proof. By Hölder's inequality, we have

$$
\begin{aligned}
|| \sigma_{\Omega, h, t}|* f(x)| & \leq\|h\|_{\Delta_{\gamma}\left(\mathbf{R}^{+}\right)}\|\Omega\|_{L^{1}\left(\mathbf{S}^{n-1}\right)}^{1 / \gamma} \\
& \times \sup _{t \in \mathbf{R}^{+}}\left(\frac{1}{t} \int_{\frac{t}{2}}^{t} \int_{\mathbf{S}^{n-1}} J\left|(y)\|\Omega(y)\| f\left(x-A_{\rho} y\right)\right|^{\gamma^{\prime}} d \sigma(y) d \rho\right)^{1 / \gamma^{\prime}} .
\end{aligned}
$$

Using Minkowski's inequality for integrals gives

$$
\begin{aligned}
\left\|\sigma_{\Omega, h}^{*} f(x)\right\|_{L^{p}\left(\mathbf{R}^{n}\right)} & \leq C\|h\|_{\Delta_{\gamma}\left(\mathbf{R}^{+}\right)}\|\Omega\|_{L^{1}\left(\mathbf{S}^{n-1}\right)}^{1 / \gamma} \\
& \times\left(\int_{\mathbf{S}^{n-1}}|\Omega(y)|\left(\left\|\mathcal{M}_{\Gamma}\left(|f|^{\gamma^{\prime}}\right)\right\|_{L^{p / \gamma^{\prime}\left(\mathbf{R}^{n}\right)}}\right) d \sigma(y)\right)^{1 / \gamma^{\prime}} .
\end{aligned}
$$

Consequently, by using Lemma 3, we finish the proof of lemma 7.

Lemma 8. Let $h \in \Delta_{\gamma}\left(\mathbf{R}^{+}\right)$for some $1<\gamma \leq 2, \Omega \in L^{q}\left(\mathbf{S}^{n-1}\right)$ for some $1<q \leq 2$ and $\theta=2^{q^{\prime} \gamma^{\prime}}$. Then for any $p$ satisfying $|1 / p-1 / 2|<1 / \gamma^{\prime}$ and $f \in L^{p}\left(\boldsymbol{R}^{n}\right)$, there is a positive constant $C_{p}$ such that

$$
\begin{gathered}
\|\left(\sum_{k \in \mathbf{Z}} \int_{\theta^{k}}^{\theta^{k+1}}\left|\sigma_{\Omega, h, t} * g_{k}\right|^{2} \frac{d t}{t} \mid\left\|_{L^{p}\left(\mathbf{R}^{n}\right)}^{\frac{1}{2}}\right\|\right. \\
\leq C_{p} \frac{\|h\|_{\Delta_{\gamma}\left(\mathbf{R}^{+}\right)}\|\Omega\|_{L^{q}\left(\mathbf{S}^{n-1}\right)}}{[(q-1)(\gamma-1)]^{1 / 2}}\left\|\left(\sum_{k \in \mathbf{Z}}\left|g_{k}\right|^{2}\right)^{1 / 2}\right\|_{L^{p}\left(\mathbf{R}^{n}\right)}
\end{gathered}
$$

holds for arbitrary functions $\left\{g_{k}(\cdot), k \in \mathbf{Z}\right\}$ on $\mathbf{R}^{n}$.

Proof. We employ some ideas from [1], [4] and [14]. By Schwarz's inequality, we obtain

$$
\begin{aligned}
\left|\sigma_{\Omega, h, t} * g_{k}(x)\right|^{2} & \leq C\|h\|_{\Delta_{\gamma}\left(\mathbf{R}^{+}\right)}^{\gamma}\|\Omega\|_{L^{q}\left(\mathbf{S}^{n-1}\right)} \\
& \times\left(\int_{\frac{1}{2} t}^{t} \int_{\mathbf{S}^{n-1}}\left|g_{k}\left(x-A_{\rho} y\right)\right|^{2}|\Omega(y)| J(y)|h(\rho)|^{2-\gamma} d \sigma(y) \frac{d \rho}{\rho}\right)
\end{aligned}
$$


Let us first prove this lemma for the case $2 \leq p<\frac{2 \gamma}{2-\gamma}$. By duality, there is a non-negative function $\psi \in L^{(p / 2)^{\prime}}\left(\mathbf{R}^{n}\right)$ with $\|\psi\|_{L^{(p / 2)^{\prime}}\left(\mathbf{R}^{n}\right)} \leq 1$ such that $\left\|\left(\sum_{k \in \mathbf{Z}} \int_{\theta^{k}}^{\theta^{k+1}}\left|\sigma_{\Omega, h, t} * g_{k}\right|^{2} \frac{d t}{t}\right)^{1 / 2}\right\|_{L^{p}\left(\mathbf{R}^{n}\right)}^{2}=\int_{\mathbf{R}^{n}} \sum_{k \in \mathbf{Z}} \int_{\theta^{k}}^{\theta^{k+1}}\left|\sigma_{\Omega, h, t} * g_{k}(x)\right|^{2} \frac{d t}{t} \psi(x) d x$. By this, equation (10) and a change of variable, we derive

$$
\begin{aligned}
& \|\left(\sum_{k \in \mathbf{Z}} \int_{\theta^{k}}^{\theta^{k+1}}\left|\sigma_{\Omega, h, t} * g_{k}\right|^{2} \frac{d t}{t}\right) \\
\leq & C\|h\|_{\Delta_{\gamma}\left(\mathbf{R}^{+}\right)}^{\gamma}\|\Omega\|_{L^{q}\left(\mathbf{S}^{n-1}\right)} \int_{\mathbf{R}^{n}}\left(\sum_{k \in \mathbf{Z}}\left|g_{k}(x)\right|^{2}\right) M_{\Omega,|h|^{2-\gamma}, \theta} \widetilde{\psi}(-x) d x,
\end{aligned}
$$

where $\widetilde{\psi}(x)=\psi(-x)$. Since $h \in \Delta_{\gamma}\left(\mathbf{R}^{+}\right)$, then $|h(\cdot)|^{2-\gamma} \in \Delta_{\frac{\gamma}{2-\gamma}}\left(\mathbf{R}^{+}\right)$, and since $\left(\frac{p}{2}\right)^{\prime}>\left(\frac{\gamma}{2-\gamma}\right)^{\prime}$, then by Lemma 7, Hölder's inequality and the same arguments used in [4], we achieve that

$$
\begin{aligned}
& \left\|\left(\sum_{k \in \mathbf{Z}} \int_{\theta^{k}}^{\theta^{k+1}}\left|\sigma_{\Omega, h, t} * g_{k}\right|^{2} \frac{d t}{t}\right)\right\|_{L^{p}\left(\mathbf{R}^{n}\right)}^{1 / 2} \\
& \leq C\|h\|_{\Delta_{\gamma}\left(\mathbf{R}^{+}\right)}^{\gamma}\|\Omega\|_{L^{q}\left(\mathbf{S}^{n-1}\right)}\left\|\left(\sum_{k \in \mathbf{Z}}\left|g_{k}\right|^{2}\right)^{1 / 2}\right\|_{L^{p}\left(\mathbf{R}^{n}\right)}^{2}\left\|M_{\Omega,|h|^{2-\gamma}, \theta} \widetilde{\psi}\right\|_{L^{(p / 2)^{\prime}\left(\mathbf{R}^{n}\right)}} \\
& \leq C \ln (\theta)\|h\|_{\Delta_{\gamma}\left(\mathbf{R}^{+}\right)}^{\gamma}\|\Omega\|_{L^{q}\left(\mathbf{S}^{n-1}\right)}\left\|\left(\sum_{k \in \mathbf{Z}}\left|g_{k}\right|^{2}\right)^{1 / 2}\right\|_{L^{p}\left(\mathbf{R}^{n}\right)}^{2}\left\|\sigma^{*}{ }_{\Omega,\left.h\right|^{2-\gamma}} \widetilde{\psi}\right\|_{L^{(p / 2)^{\prime}}\left(\mathbf{R}^{n}\right)} \\
& \leq C_{p} \frac{\|h\|_{\Delta_{\gamma}\left(\mathbf{R}^{+,}, \frac{d t}{t}\right)}^{2}\|\Omega\|_{L^{q}\left(\mathbf{S}^{n-1}\right)}^{2}}{(q-1)(\gamma-1)}\left\|\left(\sum_{k \in \mathbf{Z}}\left|g_{k}\right|^{2}\right)^{1 / 2}\right\|_{L^{p}\left(\mathbf{R}^{n}\right)}^{2} .
\end{aligned}
$$

For the case $\frac{2 \gamma}{3 \gamma-2}<p<2$; by the duality, there are functions $\zeta=\zeta_{k}(x, t)$ defined on $\mathbf{R}^{n} \times \mathbf{R}^{+}$with \|\|$\left\|\zeta_{k}\right\|_{L^{2}\left(\left[\theta^{k}, \theta^{k+1}\right], \frac{d t}{t}\right)}\left\|_{l^{2}}\right\|_{L^{p^{\prime}\left(\mathbf{R}^{n}\right)}} \leq 1$ such that 


$$
\begin{aligned}
& \left\|\left(\sum_{k \in \mathbf{Z}} \int_{\theta^{k}}^{\theta^{k+1}}\left|\sigma_{\Omega, h, t} * g_{k}\right|^{2} \frac{d t}{t}\right)\right\|_{L^{p}\left(\mathbf{R}^{n}\right)}^{\frac{1}{2}}=\int_{\mathbf{R}^{n}} \sum_{k \in \mathbf{Z}} \int_{\theta^{k}}^{\theta^{k+1}}\left(\sigma_{\Omega, h, t} * g_{k}(x)\right) \zeta_{k}(x, t) \frac{d t}{t} d x \\
& \leq C_{p} \frac{\left\|(\Upsilon(\zeta))^{1 / 2}\right\|_{L^{p^{\prime}}\left(\mathbf{R}^{n}\right)}}{[(q-1)(\gamma-1)]^{1 / 2}}\left\|\left(\sum_{k \in \mathbf{Z}}\left|g_{k}\right|^{2}\right)^{1 / 2}\right\|_{L^{p}\left(\mathbf{R}^{n}\right)},
\end{aligned}
$$

where

$$
\Upsilon \zeta(x)=\sum_{k \in \mathbf{Z}} \int_{\theta^{k}}^{\theta^{k+1}}\left|\sigma_{\Omega, h, t} * \zeta_{k}(x, t)\right|^{2} \frac{d t}{t} .
$$

As $\frac{p^{\prime}}{2}>1$, then by applying the above procedure, we reach that

$$
\begin{aligned}
\|\Upsilon(\zeta)\|_{L^{\left(p^{\prime} / 2\right)}\left(\mathbf{R}^{n}\right) \leq} \leq & C h\left\|_{\Delta_{\gamma}\left(\mathbf{R}^{+}\right)}^{\gamma}\right\| \Omega\left\|_{L^{q}\left(\mathbf{S}^{n-1}\right)}\right\|\left(\sum_{k \in \mathbf{Z}} \int_{\theta^{k}}^{\theta^{k+1}}\left|\zeta_{k}(\cdot, t)\right|^{2} \frac{d t}{t}\right) \|_{L^{\left(p^{\prime} / 2\right)\left(\mathbf{R}^{n}\right)}} \\
& \times\left\|\sigma_{\Omega,|h|^{2-\gamma}(\varsigma)}^{*}\right\|_{L^{\left(p^{\prime} / 2\right)^{\prime}}\left(\mathbf{R}^{n}\right)} \\
\leq & C\|h\|_{\Delta_{\gamma}\left(\mathbf{R}^{+}\right)}^{2}\|\Omega\|_{L^{q}\left(\mathbf{S}^{n-1}\right)}
\end{aligned}
$$

where $\varsigma$ is a function in $L^{\left(p^{\prime} / 2\right)^{\prime}}\left(\mathbf{R}^{n}\right)$ with $\|\varsigma\|_{L^{\left(p^{\prime} / 2\right)^{\prime}\left(\mathbf{R}^{n}\right)}} \leq 1$. Thus, by equations (11) and (12), our estimate holds for $\frac{2 \gamma}{3 \gamma-2} \leq p<2$; and therefore the proof of Lemma 8 is complete.

In the same manner, we achieve the following lemma.

Lemma 9. Let $h \in \Delta_{\gamma}\left(\mathbf{R}^{+}\right)$for some $\gamma \geq 2, \Omega \in L^{q}\left(\mathbf{S}^{n-1}\right)$ for some $1<q \leq 2$ and $\theta=2^{q^{\prime} \gamma^{\prime}}$. Then for any $p \in(1, \infty)$ and $f \in L^{p}\left(\boldsymbol{R}^{n}\right)$, there exists a constant $C_{p}$ such that

$$
\begin{gathered}
\|\left(\sum_{k \in \mathbf{Z}} \int_{\theta^{k}}^{\theta^{k+1}}\left|\sigma_{\Omega, h, t} * g_{k}\right|^{2} \frac{d t}{t} \mid\left\|_{L^{p}\left(\mathbf{R}^{n}\right)}^{\frac{1}{2}}\right\|_{\Delta_{p}} \frac{\gamma^{1 / 2}\|h\|_{\Delta_{\gamma}\left(\mathbf{R}^{+}\right)}\|\Omega\|_{L^{q}\left(\mathbf{S}^{n-1}\right)}}{(q-1)^{1 / 2}}\left\|\left(\sum_{k \in \mathbf{Z}}\left|g_{k}\right|^{2}\right)^{1 / 2}\right\|_{L^{p}\left(\mathbf{R}^{n}\right)}^{2}\right. \\
\leq C_{p}
\end{gathered}
$$

holds for arbitrary functions $\left\{g_{k}(\cdot), k \in \mathbf{Z}\right\}$ on $\mathbf{R}^{n}$. 


\section{Proof of Theorem 1}

We prove Theorem 1 by applying the same approaches that Al-Qassem [1] as well as Fan and Pan [14] used. Let us first assume that $h \in \Delta_{\gamma}\left(\mathbf{R}^{+}\right)$for some $1<\gamma \leq 2$. Then by Minkowski's inequality, we get that

$$
\begin{aligned}
\mathcal{M}_{\Omega, h}^{\tau} f(x) & =\left(\int_{0}^{\infty}\left|\sum_{k=0}^{\infty} t^{-\tau} \int_{2^{-k-1} t<\rho(u) \leq 2^{-k} t} f(x-u) \frac{\Omega(u) h(\rho(u))}{\rho(u)^{\alpha-\tau}} d u\right|^{2} \frac{d t}{t}\right) \\
& \leq \sum_{k=0}^{\infty}\left(\left.\int_{0}^{\infty}\left|t^{-\tau} \int_{2^{-k-1} t<\rho(u) \leq 2^{-k} t} f(x-u) \frac{\Omega(u) h(\rho(u))}{\rho(u)^{\alpha-\tau}} d u\right|^{2} \frac{d t}{t}\right|^{1 / 2}\right. \\
& =\frac{2^{a}}{2^{a}-1}\left(\int_{0}^{\infty}\left|t^{-\tau} \int_{1 / 2 t<\rho(u) \leq t} f(x-u) \frac{\Omega(u) h(\rho(u))}{\rho(u)^{\alpha-\tau}} d u\right|^{2} \frac{d t}{t}\right)^{(13)}
\end{aligned}
$$

Take $\theta=2^{q^{\prime} \gamma^{\prime}}$, and choose a $C_{0}^{\infty}(\mathbf{R})$ function, $0 \leq \varphi \leq 1$, satisfying supp $\varphi \subset\left\{y:\left[1 /\left(2 q^{\prime} \gamma^{\prime}\right) \leq \rho(y)<\left(2 q^{\prime} \gamma^{\prime}\right)\right\}\right.$, and $\sum_{k} \varphi\left(\theta^{k} \rho(x)\right)=1, x \in \mathbf{R}^{n} \backslash$ $\{0\}$. Define $\Psi_{k} \in C^{\infty}\left(\mathbf{R}^{n}\right)$ by $\widehat{\Psi_{k}}(\xi)=\varphi\left(\theta^{k} \rho(\xi)\right)$. Decompose $\sigma_{\Omega, h, t} * f(x)=$ $\sum_{j \in \mathbf{Z}} Y_{\Omega, h, j},(x, t)$, where

$$
Y_{\Omega, h, j}(x, t)=\sum_{k \in \mathbf{Z}} \sigma_{\Omega, h, t} * \Psi_{k+j} * f(x) \chi_{\left[\theta^{k}, \theta\right.}^{k+1)}(t)
$$

Define $S_{\Omega, h, j}(f)(x)=\left(\int_{0}^{\infty}\left|Y_{\Omega, h, j}(x, t)\right|^{2} \frac{d t}{t}\right)^{1 / 2}$. Then for any $f \in \mathcal{S}\left(\mathbf{R}^{n}\right)$,

$$
\left(\int_{0}^{\infty}\left|\sigma_{\Omega, h, t} * f(x)\right|^{2} \frac{d t}{t}\right) \leq \sum_{j \in \mathbf{Z}} S_{\Omega, h, j}(f)(x) .
$$

Let us first compute the $L^{2}$-norm of $S_{\Omega, h, j}(f)$. By using Plancherel's theorem, Lemma 6 and the process used in [10], we obtain that

$$
\begin{aligned}
\left\|S_{\Omega, h, j}(f)\right\|_{L^{2}\left(\mathbf{R}^{n}\right)}^{2} & \leq \sum_{k \in \mathbf{Z}_{\Gamma_{k+j}}} \int\left(\left.\int_{\theta^{k}}^{\theta^{k+1}}\left|\hat{\sigma}_{\Omega, h, t}(\xi)\right|^{2} \frac{d t}{t}|| \hat{f}(\xi)\right|^{2} d \xi\right. \\
& \leq C_{p}(\ln \theta)\|h\|_{\Delta_{\gamma}\left(\mathbf{R}^{+}\right)}^{2}\|\Omega\|_{L^{q}\left(\mathbf{S}^{n-1}\right)}^{2} 2^{-\varepsilon|j|} \sum_{k \in \mathbf{Z}} \int_{\Gamma_{k+j}}|\hat{f}(\xi)|^{2} d \xi
\end{aligned}
$$




$$
\leq C_{p}(\ln \theta)\|h\|_{\Delta_{\gamma}\left(\mathbf{R}^{+}\right)}^{2}\|\Omega\|_{L^{q}\left(\mathbf{S}^{n-1}\right)}^{2} 2^{-\varepsilon|j|}\|f\|_{L^{2}\left(\mathbf{R}^{n}\right)}^{2}
$$

for some $0<\varepsilon<1$, where $\Gamma_{k+j}=\left[\theta^{-(j+k+1)}, \theta^{-(j+k-1)}\right]$. Thus,

$$
\left\|S_{\Omega, h, j}(f)\right\|_{L^{2}\left(\mathbf{R}^{n}\right)} \leq C_{p} \frac{\|h\|_{\Delta_{\gamma}\left(\mathbf{R}^{+}\right)}\|\Omega\|_{L^{q}\left(\mathbf{S}^{n-1}\right)}}{[(q-1)(\gamma-1)]^{1 / 2}} 2^{\frac{-\varepsilon|j|}{2}}\|f\|_{L^{2}\left(\mathbf{R}^{n}\right)} .
$$

Now, let us compute the $L^{p}$-norm of $S_{\Omega, h, j}(f)$ for any $p$ satisfying $\left|\frac{1}{p}-\frac{1}{2}\right|<\frac{1}{\gamma^{\prime}}$ with $p \neq 2$. Applying Lemma 5 plus Lemma 8 , we obtain

$$
\left\|S_{\Omega, h, j}(f)\right\|_{L^{p}\left(\mathbf{R}^{n}\right)} \leq C_{p} \frac{\|h\|_{\Delta_{\gamma}\left(\mathbf{R}^{+}\right)}\|\Omega\|_{L^{q}\left(\mathbf{S}^{n-1}\right)}}{[(q-1)(\gamma-1)]^{1 / 2}}\|f\|_{L^{p}\left(\mathbf{R}^{n}\right)} .
$$

By interpolation between (15) and (16) we reach that there exists a constant $0<\mu<1$ such that

$$
\left\|S_{\Omega, h, j}(f)\right\|_{L^{p}\left(\mathbf{R}^{n}\right)} \leq C_{p} 2^{\frac{-\mu|j|}{2}} \frac{\|h\|_{\Delta_{\gamma}\left(\mathbf{R}^{+}\right)}\|\Omega\|_{L^{q}\left(\mathbf{S}^{n-1}\right)}}{[(q-1)(\gamma-1)]^{1 / 2}}\|f\|_{L^{p}\left(\mathbf{R}^{n}\right)} .
$$

Consequently, by (13), (14) and (17), we get our result for the case $h \in \Delta_{\gamma}\left(\mathbf{R}^{+}\right)$ for some $1<\gamma \leq 2$.

The proof of our theorem for the case $h \in \Delta_{\gamma}\left(\mathbf{R}^{+}\right)$for some $\gamma \geq 2$ is obtained by following the above argument except, we need to invoke Lemma 9 instead of Lemma 8. Therefore, the proof of Theorem 1 is complete.

\section{References}

[1] H. Al-Qassem, A. Al-Salman, A note on Marcinkiewicz integral operators, J. Math. Anal. Appl., 282 (2003), 698-710.

[2] H. Al-Qassem, A. Al-Salman, Rough Marcinkiewicz Integrals Related to Surfaces of Revolution, Asian J. Math., 7 (2003), 19-230.

[3] H. Al-Qassem, Y. Pan, $L^{p}$ estimates for singular integrals with kernels belonging to certain block spaces, Rev. Mat. Iberoamericana, 18 (2002), 701-730.

[4] H. Al-Qassem, Y. Pan, On certain estimates for Marcinkiewicz integrals and extrapolation, Collec. Math., 60 (2009), 123-145. 
[5] A. Al-Salman, On Marcinkiewicz integrals along flat surfaces, Turk. J. Math., 29 (2005), 111-120.

[6] Y. Chen, Y. Ding, $L^{p}$ Bounds for the parabolic Marcinkiewicz integral with rough kernels, J. Korean Math. Soc., 44 (2007), 733-745.

[7] Y. Chen, Y. Ding, A parabolic singular integral operator with rough kernel, J. Aust. Math. Soc., 84, (2008), 163-179.

[8] Y. Chen, Y. Ding, The parabolic Littlewood-Paley operator with Hardy space kernels, Canad. Math. Bull., 52 (2009), 521-534.

[9] J. Chen, D. Fan, Y. Pan, A note on a Marcinkiewicz integral operator, Math. Nachr., 227 (2001), 33-42.

[10] D. Chen, S. Lu, $L^{p}$ boundedness for parabolic Littlewood-Paley operators with rough kernels belonging to block spaces, Acta Math. Sin. (Engl. Ser.), 26 (2010), 277-286.

[11] Y. Ding, D. Fan, Y. Pan, On the $L^{p}$-boundedness of Marcinkiewicz integrals with Hardy space function kernels, Acta Math. Sin. (Engl. Ser.), 16 (2000), 593-600.

[12] J. Duoandikoetxea, J. Rubio de Francia, Maximal and singular integral operators via Fourier transform estimates, Invent. Math., 84 (1986), 541561.

[13] E. Fabes, N. Rivière, Singular integrals with mixed homogeneity, Studia Math., 27 (1966), 19-38.

[14] D. Fan, Y. Pan, Singular integral operators with rough kernels supported by subvarieties, Amer. J. Math., 119 (1997), 799-839.

[15] W. Madych, On Littlewood-Paley functions, Studia Math., 50 (1974), 4363.

[16] A. Nagel, N. Rivière, S. Wainger, On Hilbert transforms along curves, II, Amer. J. Math. 98 (1976), 395-403.

[17] F. Ricci, E. Stein, Multiparameter singular integrals and maximal functions, Ann. Inst. Fourier (Grenoble), 42 (1992), 637-670.

[18] E. Stein, Harmonic Analysis: Real-Variable Methods, Orthogonality and Oscillatory Integrals, Princeton University Press, Princeton, NJ, (1993). 
[19] F. Wang, Y. Chen, W. Yu, $L^{p}$ Bounds for the parabolic Littlewood-Paley operator associated to surfaces of revolution, Bull. Korean Math. Soc., 29 (2012), 787-797.

[20] Q. Xue, Y. Ding, K. Yabuta, Parabolic Littlewood-Paley $g$-function with rough kernels, Acta Math. Sin. (Engl. Ser.), 24 (2008), 2049-2060. 
